\title{
Stefan Storrie (ed.) (2018). Berkeley's Three Dialogues: New Essays. Oxford (UK): Oxford University Press. 240 pp.
}

Los Dialogues de Berkeley fueron publicados en mayo de 1713 en Londres. El propósito de esta obra fue clarificar algunos argumentos presentados anteriormente en la obra Treatise concerning the Principles of Human Knowledge I (1710), debido a que no fue tan bien recibida por los círculos académicos y letrados de la época. ${ }^{1}$ Contrario a lo que uno podría pensar al leer lo que acabo de mencionar, los Dialogues no son una simple iteración de lo planteado tres años antes, sino que constituyen, junto con An Essay towards a New Theory of Vision (1709), una de las tres obras centrales de la propuesta inmaterialista de Berkeley. De hecho, los diálogos presentan, respecto a los Principles, i) un nuevo estilo literario, ii) un afán por dirigirse a un público más amplio, iii) una estrategia argumentativa distinta, compleja y unificada, y iv) la omisión de algunos temas por considerar que requerirían de tecnicismos impropios de un diálogo (como la Introducción sobre las ideas abstractas). A pesar de lo anterior, y de que la obra fue muy bien recibida a lo largo del siglo XVIII, ${ }^{2}$ al grado de ser publicada cinco veces y traducida al francés en 1750 y al alemán en 1756 y en 1781, la interpretación dominante durante el siglo XX sostuvo que los Dialogues era un texto menor porque se trataba de una mera explicación dialogada de los Principles. Esta interpretación, defendida incluso por el editor de las obras completas de Berkeley, A. A. Luce, es errónea y es importante corregirla, idea que comparten los distintos ensayos que conforman Berkeley's Three Dialogues: New Essays. Sobre esto mismo el propio editor del volumen menciona lo siguiente: "La opinión actual de los especialistas de Berkeley [...] es que los Dialogues es una obra más madura en donde Berkeley desarrolla sus opiniones luego de tres años de exposición a la crítica y de una mayor reflexión sobre su posición filosófica. Muchos artículos en el presente

1 Un ejemplo de la mala comprensión que generaron los Principles es la reseña en la revista Mémoires pour l'Historie des Sciecies $\mathcal{E}$ des Beaux Arts o Mémoires de Trévoux, que considera a Berkeley un "malebranchista de buena fe" y describe su filosofía como "más allá del sentido común" (Mémoires de Trévoux, mayo de 1713, París: 921-922). Sobre la recepción de los Dialogues en la época de su publicación, véase Bracken, 1959 y McCracken y Tipton, 2000.

2 Un ejemplo de la buena opinión que suscitaron los Dialogues en muchos lectores es la carta que John Percival le envió a Berkeley el 18 de julio de 1713. (Cfr. Hight, 2013: 97-99). 
volumen muestran buenas razones para pensar que la opinión de Berkeley evolucionó y se complejizó de distintas maneras. El presente volumen, que es la primera colección de artículos sobre los Dialogues, llega en un gran momento para los estudios berkeleyanos" (p. 4).

Una vez dicho lo anterior, es momento de centrarse en el libro Berkeley's Three Dialogues: New Essays, editado por Stefan Storrie, joven profesor sueco que ha laborado en el Trinity College de Dublín, alma mater de Berkeley, y que representa la nueva generación de estudiosos sobre Berkeley. Este hecho explica, en parte, que los participantes en el libro sean tanto jóvenes berkeleyanos como algunos de los más destacados especialistas en el filósofo irlandés.

El libro consta de doce artículos antecedidos por una introducción del propio Storrie, en donde menciona algunas cosas importantes sobre la nueva publicación, dentro de las cuales destaco dos: i) la que subraya el hecho de que a pesar de haberse publicado varios libros sobre los Dialogues, este nuevo volumen tiene la peculiaridad de ser el primero en estar constituido por un conjunto de ensayos dedicados exclusivamente a aquella obra (lo que lo hace especialmente relevante porque, a diferencia de las otras publicaciones, brinda un panorama amplio, completo y más variado sobre el texto berkeleyano). ii) Lo segundo a destacar tiene que ver tanto con el contenido como con el enfoque del libro, cuyo objetivo es, como el propio editor aclara, "examinar asuntos que Berkeley plantea en los Dialogues. Esto no significa que los artículos versen exclusivamente sobre esta obra, sino que más bien este volumen toma los Dialogues como punto de partida" (p. 4).

El volumen que aquí se reseña tiene la virtud de que los artículos que lo integran toman como base los Dialogues, pero no se olvidan de obras tan importantes como los Principles, la New Theory of Vision (1710), De Motu (1721), Siris (1744) o el bien conocido Alciphron (1732). Esto es importante porque a pesar de tratarse de estudios especializados, no caen en el error de hacer que sus textos sean tan específicos que resulten ininteligibles para los no iniciados; por el contrario, los autores se basan en los diálogos de 1713 pero no olvidan interrelacionarlos con otros textos, de ahí que los diversos artículos resulten amenos e interesantes para todo interesado en Berkeley y en la filosofía moderna en general. Incluso como los propios artículos evidencian el tipo de aproximación que dan, fue fácil para el editor organizar "los primeros ocho artículos [...] para seguir en líneas generales la estructura general de los diálogos; 
los últimos cuatro trabajos consideran la obra en su contexto filosófico más amplio" (p. 4).

Como se desprende del párrafo anterior, el libro está conformado por doce artículos que versan sobre problemas epistemológicos, ontológicos, perceptuales, religiosos y morales. El primer artículo, que formalmente constituye el capítulo dos del libro, es escrito por Lisa Downing, profesora en la Universidad Estatal de Ohio. Su artículo se titula Sensible Qualities and Secondary Qualities in the First Dialogue y estudia el cambio que Berkeley implementó en su terminología ontológica, al sustituir la palabra 'ideas', usada en los Principles, por 'cualidades sensibles', empleada en los Dialogues. Downing, quien reconoce que la obra de 1713 ha sido "subestimada" al igual que su propio autor (p. 7), argumenta que la concepción de "idea" pertenece principalmente a la teoría representacionista de la filosofía de la percepción, mientras que el uso de la expresión "cualidad sensible" lleva a la clásica distinción lockeana entre cualidades primarias y secundarias, y, sobre todo, a los fundamentos metafísicos de la "nueva filosofía" mecanicista. La comentarista arguye que la obra Principles tiene objetivos distintos al primero de los tres diálogos, pues mientras aquélla ataca el mecanicismo materialista "vía su teoría representativa de la percepción", los Dialogues atacan el mecanicismo materialista "vía su distinción entre cualidades primarias/secundarias" (p. 7).

Los dos artículos siguientes se relacionan con el de Downing porque consideran, más en detalle, asuntos de percepción en los Dialogues. El tercer artículo del libro corre a cargo de Thomas Stoneham, actual vicepresidente de la 'International Berkeley Society' y profesor en la Universidad de York, Inglaterra. Su artículo, Some Issues in Berkeley's Account of Sense Perception, sostiene que la manera correcta de interpretar cómo comprende Berkeley los objetos del sentido común, es reconociendo que para percibir el objeto no necesitamos percibir todas las ideas que lo constituyen; aunque el tema que aborda no es un asunto nuevo, el análisis de Stoneham es sutil e incluye una revisión interesante de dos teorías recientes (de Georges Dicker, 2011, y de George Pappas, 2000) sobre la percepción inmediata en Berkeley. En suma, la lectura de Stoneham señala que al atribuirle al irlandés una postura filosófica más razonable, a saber, que la percepción de un objeto del sentido común, como una silla, no requiere que percibamos todas las ideas que constituyen el objeto, se puede comprender mejor el inmaterialismo berkeleyano. 
El cuarto artículo, Berkeley on the Objects of Perception, es de Jennifer Smalligan Marušić, profesora en la Universidad Brandeis en Estados Unidos. El artículo de Marušić, que como su nombre indica se centra en los objetos de la percepción, plantea la teoría alternativa de la "percepción del acto-objeto", la cual sostiene que hay dos aspectos en la percepción: i) una sensación, que es un acto de la mente, y ii) un objeto inmediatamente percibido. Marušić arguye que la posición de Berkeley respecto a la percepción debe entenderse en el sentido de que las cualidades sensibles son objetos de percepción, dependientes de la mente, y no actos perceptivos.

El artículo siguiente muestra un cambio temático respecto a los anteriores. Se trata del texto de Keota Fields, profesor en la Universidad de Massachusetts Darmouth. Su escrito se titula Berkeley's Semiotic Idealism y versa sobre el estatuto ontológico de los objetos sensibles. Fields plantea un aparente conflicto en la explicación de Berkeley de los objetos sensibles, pues por un lado los describe como conjuntos de experiencias subjetivas $\mathrm{y}$, por otro, como objetos estables y continuos que existen en la mente de Dios. El comentarista arguye que para Berkeley los objetos sensibles son "compuestos de ideas internas y divinas cuya percepción es prescrita por reglas universales" (p. 82), es decir, propone ver estos dos elementos entrelazados por significados prescritos por normas lingüísticas universales; de ahí que concluya en su artículo que la relación semiótica da forma y significado objetivo a las ideas subjetivas.

El siguiente texto corre a cargo de Samuel Rickless, profesor de la Universidad de California en San Diego. Su artículo, Berkeley's Argument for the Existence of God in the Three Dialogues, presenta por un lado un análisis detallado de los argumentos para la existencia de Dios presentes en los Dialogues, y, por otro, compara dichos argumentos con los que Berkeley desarrolla en los Principles. Rickless, interesado en revisar analíticamente los argumentos berkeleyanos, arguye en relación al tema que la argumentación presente en la obra dialogada es más detallada y explícita que la desarrollada en 1710, siendo un ejemplo de ello la imposibilidad del agente ciego y la estabilidad que se observa en el mundo sensible.

Tras el texto de Rickless sigue el artículo de Sukjae Lee, profesor de la Universidad Nacional de Seúl, Corea, titulado Berkeley on Continuous Creation: Occasionalism Contained. Este artículo analiza en detalle la idea de Dios como creación continua del mundo, asunto implícito en muchas 
de las metafísicas de la temprana modernidad y que fue una característica central del ocasionalismo de Malebranche, de quien Lee se sirve para su escrito. Para el comentarista coreano el compromiso de Berkeley con el ocasionalismo es parcial y "limitado", pues mientras el irlandés asumió que los cuerpos físicos (ideas) eran creados continuamente por Dios, no creyó lo mismo respecto a los espíritus o mentes; más aún, argumenta que la idea de creación continua no es para Berkeley un punto de partida metafísico, sino una explicación útil y consistente con una amplia gama de fenómenos.

El octavo artículo, The Active Self and Perception in Berkeley's Three Dialogues, es de James Hill, profesor en la Universidad Karlova de Praga. En su escrito Hill estudia el problema del autoconocimiento del yo y subraya tres aspectos del planteamiento berkeleyano que considera problemáticos, a partir de "la opinión de Berkeley de que un yo activo es el sujeto de la percepción cuando la percepción misma se interpreta como completamente pasiva" (p. 123). El primer aspecto problemático tiene que ver con el hecho de que para Berkeley la mente es esencialmente activa por ser volitiva. Esto la hace completamente distinta de las ideas o cosas, por lo que el conocimiento de la mente es muy distinto al de los objetos sensibles. El segundo aspecto es que cuando percibimos ideas somos completamente pasivos. El último se enfoca en que la mente es un ser simple e indivisible y por lo mismo no puede ser tanto activa como pasiva. Desde la perspectiva de Hill, Berkeley niega el segundo aspecto, lo que lo lleva a considerar que "la mente une activamente las percepciones pasivas de diferentes modalidades sensibles en objetos de la experiencia" (p. 134); de ahí que concluya que la mente es permanentemente activa.

El noveno escrito es de Stephen Daniel, expresidente de la 'International Berkeley Society' y actual profesor de la Universidad de Texas A\&M. Su artículo se intitula Berkeley on God's Knowledge of Pain y versa sobre la manera en que Dios conoce el mundo a partir del hecho de que no puede sentir, lo que implica pasividad e imperfección; a partir de esto Daniel se pregunta, ¿cómo puede conocer Dios sensaciones como las de dolor? El comentarista arguye que Dios comprehende la realidad como un todo armonioso, mientras nuestra percepción de dolor es la percepción de cierto tipo de disarmonía. El dolor, pues, no es tanto una idea que se interrumpa como una forma de percibir una relación entre ideas. Dios, concluye Daniel, percibe todas las ideas que nosotros 
percibimos, pero entiende su relación de una manera distinta a como nosotros lo hacemos.

Los últimos cuatro artículos del libro, menciona Storrie, no estudian propiamente los Dialogues, sino que se sirven de ellos para tratar asuntos filosóficos más amplios. El décimo artículo es elaborado por John Russell Roberts, profesor de la Universidad Estatal de Florida, y se titula A Puzzle in the Three Dialogues and Its Platonic Resolution. Roberts se pregunta cómo es que Berkeley, en su obra dialogada, acepta tanto la facultad del "intelecto puro" como las ideas innatas pero rechaza la facultad de abstraer. La solución que ofrece el comentarista, y que relaciona estrechamente con la obra Siris (1744), es presentar una lectura neoplatónica de Berkeley, quien fue influido -según Roberts- por el neoplatónico Ralph Cudworth, en la que las ideas innatas del intelecto puro son principios activos constitutivos del yo.

El siguiente artículo es del propio editor y se titula The Scope of Berkeley's Idealism in the 1734 Edition of the Three Dialogues. En su escrito Storrie analiza cómo es que Berkeley llega a afirmar su ontología, i.e., que sólo hay mentes e ideas; para tratar el asunto aborda desde los Notebooks (1707-08) hasta la segunda edición de los Dialogues (1734), con el propósito de ver cómo se desarrolló la propia concepción de Berkeley del idealismo. Storrie señala cuatro "posibilidades" en relación a lo que Berkeley pudo querer decir cuando hablaba de su principio inmaterialista (p. 162), y concluye atribuyendo a Berkeley la opinión de que su principio inmaterialista es como una hipótesis científica, pues puede ser falseado por evidencia empírica pero no es susceptible de una "prueba" empírica. En la opinión de Storrie esto explica muy bien por qué el irlandés retoma el concepto de espacio absoluto, ya que piensa que si éste existe (y si ello puede mostrarse razonablemente) ello socavaría sus principios inmaterialistas; empero, considera Storrie, Berkeley piensa que los argumentos para el espacio absoluto fallan y en ello se apoya para defender la factibilidad de su teoría filosófica.

Kenneth L. Pearce, joven profesor del Trinity College de Dublín, escribe el penúltimo artículo: Matter, God and Nonsense: Berkeley's Polemic against the Freethinkers in the Three Dialogues. ${ }^{3}$ En él intenta resolver una posible tensión en Berkeley, quien en algunas notas de los Philosophical

3 Pearce ya trató este tema en su artículo "Berkeley's Philosophy of Religion" (2017): 458-83. 
Commentaries (denominados Notebooks ${ }^{4}$ por muchos estudiosos) se dirige explícitamente a los librepensadores mientras menciona que no debe ofender a los eclesiásticos. ¿Por qué se preocuparía por ofender a los eclesiásticos mientras defendía a la Iglesia de los librepensadores?, se pregunta el comentarista. Su respuesta, para la cual retoma argumentos del Alciphron, es que para entender el tipo de ataque de Berkeley se deben revisar algunas partes de la teología filosófica, relacionadas con la analogía divina. Berkeley -arguye Pearce- piensa que la fe común del vulgo es suficiente para refutar los desafíos ateos, aunque puede llegar a ofender la sensibilidad de los teólogos. Desarrollar este asunto es el objetivo del escrito.

El último artículo, Hey, What's the Big Idea? Berkeley and Hume on Extension, Local Conjunction, and the Immateriality of the Soul, es de Don Garrett, profesor en la Universidad de Nueva York. Garrett, como estudioso de Berkeley y Hume, aborda en su artículo el concepto de idea de los dos empiristas y contrapone la tesis de Berkeley sobre las ideas en la mente con la teoría humeana del yo. El autor argumenta que ambos filósofos comparten un compromiso real con la existencia de ideas extensas o percepciones, de ahí que explore en su artículo las diferentes estrategias que utiliza cada uno para explicar cómo la mente puede incluir tanto cosas extensas como inextensas. Conviene señalar que Garrett es cuidadoso al indicar que el hecho de que algunas ideas sean extensas, no implica que la percepción lo sea o que tales ideas deban localizarse espacialmente (p. 196).

El libro editado por Storrie es un compendio de doce artículos académicos, especializados, que giran en torno a los Dialogues. Cada artículo contribuye a los estudios sobre Berkeley pero también a los de la filosofía moderna, en la medida en que los planteamientos de un filósofo remiten a los de otro y eso obliga a relacionar unas teorías con otras. Debo mencionar, sin embargo, que ningún artículo del volumen Berkeley's Three Dialogues: New Essays desarrolla una tesis o interpretación absolutamente innovadora, es decir, nunca planteada en la literatura secundaria. Esto no significa que el volumen no aporte nada, pues

$4 \quad$ El debate de los años noventa sobre cómo nombrar las anotaciones berkeleyanas de 1707-08, trajo como resultado que la mayoría de los especialistas hablen de 'Cuadernos' y no de 'Comentarios filosóficos', pues, arguyen, el término 'comentario filosófico' remite a reflexiones claras y precisas, algo que no se corresponde con las anotaciones. 
porque no se trate de una obra revolucionaria no se sigue que no sea valiosa; por el contrario, cada uno de los textos brinda planteamientos y enfoques interesantes sobre los temas que trata, lo que enriquece la discusión y la mejor comprensión de los Dialogues. Más aún, se trata de un libro útil y refrescante para todos los especialistas en Berkeley y los interesados en su pensamiento, debido a que cada uno de los artículos analiza a profundidad, sirviéndose de la literatura reciente, los argumentos centrales de los Dialogues y lo hace-y eso es importante- de manera seria, rigurosa y bien fundamentada.

\section{Bibliografía}

Bracken, Harry M. (1959). The Early Reception of Berkeley's Immaterialism 1710-1733. The Hague: Martinus Nijhoff.

Hight, M. A. (ed.) (2013). The Correspondence of George Berkeley. Nueva York: Cambridge University Press.

McCracken, C.; Tipton, I. C. (2000). Berkeley's Principles and Dialogues: Background Source Materials. Nueva York: Cambridge University Press.

Pearce, K. (2017). Berkeley's Philosophy of Religion. En R. Brook, B. Belfrage (eds.), The Bloomsbury Companion to Berkeley. Nueva York: Bloomsbury Academic.

Alberto Luis López Universidad Nacional Autónoma de México albertograco@yahoo.com.mx 